\title{
IGNITION OR NONIGNITION OF A COMBUSTIBLE SOLID WITH MARGINAL HEATING
}

\author{
BY \\ D. GLENN LASSEIGNE (Old Dominion University, Norfolk, Virginia) \\ AND \\ W. E. OLMSTEAD (Northwestern University, Evanston, Illinois)
}

\begin{abstract}
The possibility of achieving ignition of a combustible solid with only marginal external heating is investigated. The heating barely raises the temperature to a level where reaction occurs. A parameter indicating the duration of time spent near the crucial level governs the ignition process. If the parameter is below a critical value, no thermal runaway can occur; if it is sufficiently large, ignition will be achieved.
\end{abstract}

1. Introduction. The problem of interest here concerns the possibility of igniting a solid combustible material when only a marginal amount of external stimulus is supplied. Since the onset of ignition takes place in a thin layer near the heated surface of the material, we model the problem by a semi-infinite slab $(x \geq 0)$ subjected to external heating at one end $(x=0)$. We formulate the problem so that this external heating only barely raises the surface temperature to a level that might produce thermal runaway. Indeed, we will identify a critical value of this heating that distinguishes between ignition and nonignition situations.

In nondimensional form, the temperature $\theta(x, t)$ of the material and the mass fraction of unexpended fuel $Y(x, t)$ satisfy the system

$$
\begin{gathered}
\theta_{t}=\theta_{x x}+A Y e^{-E / \theta}, \quad x>0, t>0, \\
Y_{t}=-B^{-1} A Y e^{-E / \theta}, \quad x>0, t>0, \\
\theta_{x}(0, t)=-h(t), \quad \theta(\infty, t)=1, \theta(x, 0)=1, \\
Y(x, 0)=1 .
\end{gathered}
$$

Both the temperature and mass fraction have been normalized by their (constant) initial values. The nondimensional parameters $A, B$, and $E$ respectively denote the Damkohler number, the heat-release constant, and the activation energy. The exponential term models a first-order, Arrhenius-type of chemical reaction; in (1.1) it represents the source of internal heating, while in (1.2) it serves to deplete the available fuel. The external stimulation of the system by the surface heat flux $h(t)$ 
will be restricted so as to only marginally excite the reaction term. The spatial diffusion term in (1.2) has been neglected because it is scaled by the reciprocal of the Lewis number which is quite large for solids.

The system (1.1)-(1.4), or some modified version, has been the subject of numerous investigations of the ignition problem. Linan and Williams [5] originally developed an asymptotic technique based on $E \gg 1$ and $A \gg 1$ for (1.1) with $Y \equiv 1$ and (1.3). For a constant heat flux $h(t) \equiv 1$, they derived an integral equation for the surface temperature which predicts the thermal runaway leading to ignition. Their analysis was extended by Kapila [3] to allow for variable $Y$ as well as to follow the evolution of the problem through post-ignition.

Olmstead [6] considered (1.1)-(1.4) with a variable heat flux $h(t)$ and found sufficient conditions under which thermal runaway would occur and be predicted by essentially the same integral equation as in [5]. In this paper, we will relax one of those sufficient conditions and derive a new integral equation which governs thermal runaway. This integral equation contains a parameter whose value depends on $h(t)$. Analysis of the integral equation reveals a critical value of this parameter which must be exceeded before thermal runaway will occur.

Our investigation here utilizes $E \gg 1$ and $A \gg 1$ in the usual way by setting

$$
A=A_{0} E^{1 / 4} \theta_{c}^{-1 / 2} e^{E / \theta_{c}},
$$

which defines a special temperature $\theta_{c}$ near which the reaction term becomes significant. We insist that $\theta_{c}>1$ so that the system (1.1)-(1.4) starts below this crucial level, with the need for some external stimulus to raise the temperature near $\theta_{c}$. The small parameter

$$
\varepsilon \equiv \theta_{c}^{2} / E \ll 1
$$

is used as the basis of the asymptotic analysis of (1.1)-(1.4).

With (1.5)-(1.6), the reaction term can be expressed as

$$
A Y e^{-E / \theta}=A_{0} Y \varepsilon^{-1 / 4} \exp \left[\varepsilon^{-1} \theta_{c}^{2}\left(\theta_{c}^{-1}-\theta^{-1}\right)\right] .
$$

Our analysis will first consider the inert stage when $\theta$ is significantly less than $\theta_{c}$ and (1.7) is exponentially small. Then we examine the transition stage when the external heating is only marginally adequate to raise $\theta$ to the level of $\theta_{c}$.

We assume here, as in [6], that the heat release parameter $B$ is $O(1)$ relative to $\varepsilon$. This results in only small changes in $Y(x, t)$ during the transition stage, with no influence on thermal runaway. It is possible to inhibit thermal runaway by scaling $B$ large enough to enhance reactant consumption, as was done in [4]; however, we do not include that effect here.

2. Inert stage and marginal heating. During the inert stage of the problem, when $\theta$ is significantly less than $\theta_{c}$, the reaction term (1.7) is exponentially small. This uncouples the system (1.1)-(1.4) into two independent and linear problems, which are solved by

$$
\begin{gathered}
\theta_{I}(x, t)=1+\int_{0}^{t}[\pi(t-s)]^{-1 / 2} \exp \left[-x^{2} / 4(t-s)\right] h(s) d s \\
Y_{I}(x, t)=1 .
\end{gathered}
$$


These inert stage solutions remain valid until such time that $\theta$ becomes comparable to $\theta_{c}$. Since $\theta_{I}$ attains its maximum value at $x=0$, the special time $t_{c}>0$ near which the reaction term becomes significant is conveniently defined by

$$
1<\theta_{c}=\theta_{I}\left(0, t_{c}\right)=1+\int_{0}^{t_{c}}\left[\pi\left(t_{c}-s\right)\right]^{-1 / 2} h(s) d s .
$$

We assume here that the properties of $h(t)$ are such that there exists some $t_{c}$ which satisfies (2.3). Moreover, if there is more than one possible value, we specify $t_{c}$ as the minimum.

The behavior of $\theta_{I}$ in the neighborhood of $\theta_{c}$ plays a crucial role as the inert stage gives way to the transition stage when the reaction term becomes important. One of the key results of [6] is the specification of

$$
a \equiv \frac{\partial \theta_{I}}{\partial t}\left(0, t_{c}\right)>0, \quad b \equiv-\frac{\partial \theta_{I}}{\partial x}\left(0, t_{c}\right)>0
$$

as sufficient conditions for the occurrence of thermal runaway. From a physical viewpoint, these conditions suggest that the external stimulus must not only drive the end-temperature above $\theta_{c}$, but also have some heat entering the system as that level is reached.

For the constant flux case (i.e., $h(t)=1$ ) as considered in [3] and [5], the conditions of (2.4) are automatically fulfilled. In [6] it was pointed out that for a variable flux these conditions need not be satisfied, and that under certain circumstances this leads to an indefinite delay of ignition.

We investigate here the circumstances of marginal heating to achieve thermal runaway by replacing (2.4) with

$$
a \equiv \frac{\partial \theta_{I}}{\partial t}\left(0, t_{c}\right)=0, \quad b \equiv-\frac{\partial \theta_{I}}{\partial x}\left(0, t_{c}\right)>0, \quad c \equiv-\frac{1}{2} \frac{\partial^{2} \theta_{I}}{\partial t^{2}}\left(0, t_{c}\right)>0 .
$$

The physical interpretation of $(2.5)$ is that the external heating provided by $h(t)$ is barely adequate to raise the end-temperature to the level of $\theta_{c}$, in the sense of tangential incidence. We retain $b>0$, the condition of having some heat entering the system as the crucial level is reached. The added condition that $c>0$ is consistent with $\theta_{I}\left(0, t_{c}\right)=\theta_{c}$ being a local maximum. It is convenient to interpret $c^{-1}$ as a measure of the duration of time that $\theta_{I}(0, t)$ remains close to $\theta_{c}$. In fact, we will find a critical value for $c$ which distinguishes between ignition and nonignition.

It should be noted that the marginal heating condition $a=0$ of (2.5) cannot be realized with constant heat flux. In fact, $h(t)$ must undergo a decrease before $t_{c}$ is reached to fulfill that condition.

3. Transition stage. As the external heating drives the temperature near $\theta_{c}$, the reaction term becomes significant. Since this first occurs close to the surface $x=0$ as $t$ nears $t_{c}$, we introduce the new independent variables

$$
\begin{gathered}
x=\varepsilon \xi, \quad \xi \geq 0, \\
t=t_{c}+\varepsilon^{1 / 2} \tau, \quad \tau \geq-\varepsilon^{-1 / 2} t_{c} \rightarrow-\infty .
\end{gathered}
$$


This change of variables together with (1.7) allows us to express (1.1) and (1.2) as

$$
\begin{aligned}
& \varepsilon^{3 / 2} \theta_{\tau}=\theta_{\xi \xi}+\varepsilon^{7 / 4} A_{0} Y \exp \left[\varepsilon^{-1} \theta_{c}^{2}\left(\theta_{c}^{-1}-\theta^{-1}\right)\right], \\
& \varepsilon^{3 / 2} Y_{\tau}=-\varepsilon^{7 / 4} B^{-1} A_{0} Y \exp \left[\varepsilon^{-1} \theta_{c}^{2}\left(\theta_{c}^{-1}-\theta^{-1}\right)\right] .
\end{aligned}
$$

We investigate the solutions of (3.2) and (3.3) as perturbations of the inert solutions. That is

$$
\begin{gathered}
\theta=\theta_{I}\left(\varepsilon \xi, t_{c}+\varepsilon^{1 / 2} \tau\right)+\varepsilon \varphi_{0}(\xi, \tau)+\varepsilon^{7 / 4} \varphi_{1}(\xi, \tau)+\cdots, \\
Y=1+\varepsilon^{1 / 4} z_{0}(\xi, \tau)+\cdots
\end{gathered}
$$

When (3.4) and (3.5) are substituted into (3.2) and (3.3), the inert solutions are eliminated by the linear operators. In order to determine their contribution to the reaction term, we note that

$$
\theta_{I}\left(\varepsilon \xi, t_{c}+\varepsilon^{1 / 2} \tau\right)=\theta_{c}-\varepsilon\left(b \xi+c \tau^{2}\right)+o(\varepsilon)
$$

and hence

$$
Y \exp \left[\varepsilon^{-1} \theta_{c}^{2}\left(\theta_{c}^{-1}-\theta^{-1}\right)\right]=\exp \left[\varphi_{0}(\xi, \tau)-b \xi-c \tau^{2}\right]+o(1) .
$$

We need only be concerned here with the perturbations of temperature. It follows that at $O(\varepsilon)$,

$$
\frac{\partial^{2} \varphi_{0}}{\partial \xi^{2}}(\xi, \tau)=0, \quad \xi>0, \tau>-\infty ; \quad \frac{\partial \varphi_{0}}{\partial \xi}(0, \tau)=0, \quad \varphi_{0}(\xi,-\infty)=0
$$

This is satisfied by

$$
\varphi_{0}(\xi, \tau)=f_{0}(t), \quad f_{0}(-\infty)=0 .
$$

Then at $O\left(\varepsilon^{7 / 4}\right)$, it follows that

$$
\begin{aligned}
& \frac{\partial^{2} \varphi_{1}}{\partial \xi^{2}}(\xi, \tau)=-A_{0} \exp \left[f_{0}(\tau)-b \xi-c \tau^{2}\right], \quad \xi>0, \tau>-\infty \\
& \frac{\partial \varphi_{1}}{\partial \xi}(0, \tau)=0, \quad \varphi_{1}(\xi,-\infty)=0 .
\end{aligned}
$$

This is satisfied by

$$
\varphi_{1}(\xi, \tau)=-\frac{A_{0}}{b}\left(\xi+\frac{1}{b} e^{-b \xi}\right) \exp \left[f_{0}(\tau)-c \tau^{2}\right]+f_{1}(\tau), \quad f_{1}(-\infty)=0 .
$$

In solving for $\varphi_{0}$ and $\varphi_{1}$, no condition as $\xi \rightarrow \infty$ has been imposed, because (3.4) is regarded as the inner solution valid in a thin zone near $x=0$ where the reaction term has become significant. These must be matched to an outer solution valid outside of the reaction zone.

To obtain the appropriate outer solution, we rescale the spatial variable as

$$
x=\varepsilon^{1 / 4} X
$$

and consider the expansion

$$
\theta=\theta_{I}\left(\varepsilon^{1 / 4} X, t_{c}+\varepsilon^{1 / 2} \tau\right)+\varepsilon \Phi_{0}(X, \tau)+\cdots .
$$


In this case the reaction term makes the form

$$
Y \exp \left[\varepsilon^{-1} \theta_{c}^{2}\left(\theta_{c}^{-1}-\theta^{-1}\right)\right]=\exp \left[-\varepsilon^{-3 / 4} b X+\Phi_{0}(X, \tau)-c \tau^{2}\right]+\cdots,
$$

which is seen to be exponentially small. Thus, it follows that

$$
\begin{aligned}
& \left(\frac{\partial}{\partial \tau}-\frac{\partial^{2}}{\partial X^{2}}\right) \Phi_{0}(X, \tau)=0, \quad X>0, \tau>-\infty ; \\
& \Phi_{0}(\infty, \tau)=0, \quad \Phi_{0}(X,-\infty)=0 .
\end{aligned}
$$

In order to have $\Phi_{0}$ properly determined, some condition at $X=0$ is needed. That condition follows by an asymptotic matching to the inner solution. From (3.4) and (3.13), we find that

$$
\begin{aligned}
\varepsilon \Phi_{0}(X, \tau)+\cdots & =\varepsilon \varphi_{0}\left(\varepsilon^{-3 / 4} X, \tau\right)+\varepsilon^{7 / 4} \varphi_{1}\left(\varepsilon^{-3 / 4} X, \tau\right)+\cdots \\
& =\varepsilon\left\{f_{0}(\tau)-\frac{A_{0}}{b} X \exp \left[f_{0}(\tau)-c \tau^{2}\right]\right\}+o(\varepsilon),
\end{aligned}
$$

and hence

$$
\begin{gathered}
\Phi_{0}(0, \tau)=f_{0}(\tau), \\
\frac{\partial \Phi_{0}}{\partial X}(0, \tau)=-\frac{A_{0}}{b} \exp \left[f_{0}(\tau)-c \tau^{2}\right] .
\end{gathered}
$$

Now the solution of (3.15) which also satisfies (3.18) is

$$
\Phi_{0}(X, \tau)=\frac{A_{0}}{b} \int_{-\infty}^{\tau}[\pi(\tau-\sigma)]^{-1 / 2} \exp \left\{f_{0}(\sigma)-c \sigma^{2}-\left[X^{2} / 4(\tau-\sigma)\right]\right\} d \sigma .
$$

Evaluation of (3.19) at $X=0$ and utilizing (3.17) gives

$$
f_{0}(\tau)=\frac{A_{0}}{b} \int_{-\infty}^{\tau}[\pi(t-\sigma)]^{-1 / 2} \exp \left[f_{0}(\sigma)-c \sigma^{2}\right] d \sigma .
$$

This nonlinear integral equation (3.20) determines $f_{0}(\tau)$, and hence $\varphi_{0}$ and $\Phi_{0}$ as seen from (3.9) and (3.19), respectively. Thus, the leading order perturbation of the inert temperature is governed by (3.20). If the solution of (3.20) becomes unbounded at some $\tau^{*}<\infty$, that is interpreted as the thermal runaway associated with ignition. This defines an ignition time,

$$
t^{*}=t_{c}+\sqrt{\varepsilon} \tau^{*}
$$

In Sec. 4 we investigate the properties of (3.20), and find that thermal runaway does not occur if $c$ exceeds some critical value. Thus, the marginal heating conditions (2.5) yield results in distinct contrast with those of (2.4). When (2.4) holds (with some minor changes in scaling), it is found that

$$
f_{0}(\tau)=\frac{A_{0}}{b} \int_{-\infty}^{\tau}[\pi(t-\sigma)]^{-1 / 2} \exp \left[f_{0}(\sigma)+a \sigma\right] d \sigma .
$$

As discussed in $[3,5,6]$, the solution of (3.22) always becomes unbounded at some $\tau^{*}<\infty$. 
4. Analysis of the integral equation. Here we examine the properties of the integral equation (3.20) which governs the perturbation of the inert temperature. Of particular interest are the circumstances which distinguish between its solution being bounded for all $\tau$ and becoming unbounded at some $\tau^{*}<\infty$.

It is convenient to introduce the new variables

$$
u(\eta)=f_{0}(\tau), \quad \eta=c^{1 / 2} \tau,
$$

and define the parameter

$$
\gamma=A_{0} b^{-1} c^{-1 / 4}>0
$$

whereupon (3.20) takes the form

$$
u(\eta)=\gamma T u(\eta) \equiv \gamma \int_{-\infty}^{\eta}[\pi(\eta-\zeta)]^{-1 / 2} \exp \left[u(\zeta)-\zeta^{2}\right] d \zeta
$$

We first investigate (4.3) for the existence of a unique, bounded solution. Since it is clear that any solution of (4.3) must be nonnegative, we consider functions $u(\eta)$ which are continuous on the interval $(-\infty, \hat{\eta})$ with $0 \leq u(\eta) \leq M$. To apply a contraction mapping argument to (4.3), we first note that

$$
\gamma T u(\eta) \leq \gamma e^{M} F(\eta)
$$

where

$$
F(\eta)=\int_{-\infty}^{\eta}[\pi(\eta-\zeta)]^{-1 / 2} e^{-\zeta^{2}} d \zeta=\int_{0}^{\infty}(\pi \zeta)^{-1 / 2} \exp \left[-(\eta-\zeta)^{2}\right] d \zeta .
$$

Thus $\gamma T$ maps the indicated space into itself whenever

$$
\gamma e^{M} F(\eta) \leq M .
$$

The contraction property is found by considering

$$
\sup _{(-\infty, \hat{\eta})}\left|\gamma T u_{1}-\gamma T u_{2}\right| \leq \gamma e^{M} F(\eta) \sup _{(-\infty, \hat{\eta})}\left|u_{1}-u_{2}\right| .
$$

This implies a contraction when

$$
\gamma e^{M} F(\eta)<1 .
$$

In order to fulfill both (4.6) and (4.8), the optimal choice is $M=1$. Then (4.3) will have a unique continuous bounded solution for all $\eta<\hat{\eta}$ provided that

$$
\gamma e F(\eta)<1, \quad-\infty<\eta<\hat{\eta} .
$$

To understand the implications of (4.9), we need more specific information about $F(\eta)$. It follows from $(4.5)$ that $F(\eta)$ satisfies

$$
\begin{aligned}
& F^{\prime \prime}(\eta)+2 \eta F^{\prime}(\eta)+F(\eta)=0, \quad-\infty<\eta<\infty, \\
& F(0)=\Gamma\left(\frac{1}{4}\right) / 2 \sqrt{\pi}, \quad F^{\prime}(0)=\Gamma\left(\frac{3}{4}\right) / \sqrt{\pi} .
\end{aligned}
$$

The desired solution of (4.10) can be expressed as

$$
F(\eta)=\frac{\sqrt{\pi}}{2}|\eta|^{1 / 2} e^{-\eta^{2} / 2}\left[I_{-1 / 4}\left(\frac{\eta^{2}}{2}\right)+I_{1 / 4}\left(\frac{\eta^{2}}{2}\right) \operatorname{sgn} \eta\right],
$$




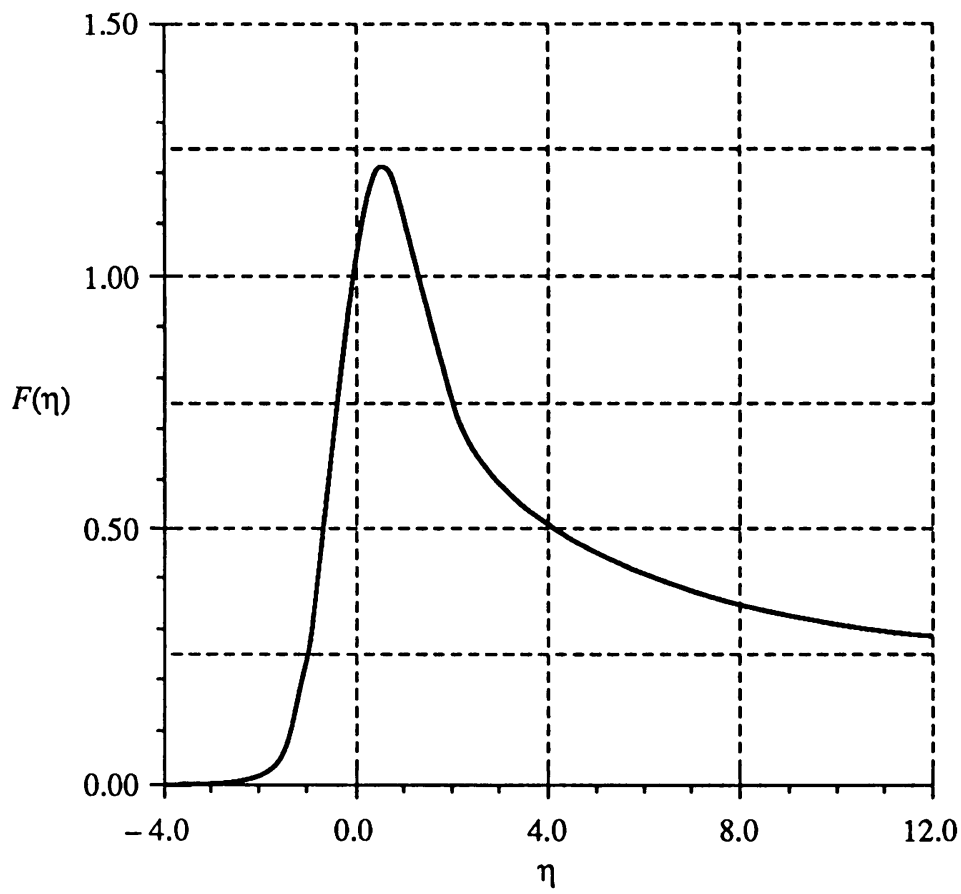

FIG. 1. $F(\eta)=\int_{-\infty}^{\eta}[\pi(\eta-\zeta)]^{-1 / 2} e^{-\zeta^{2}} d \zeta$. The maximum value $F(\bar{\eta}) \approx 1.214$ occurs at $\bar{\eta} \approx 0.541$.

where $I_{1 / 4}$ and $I_{-1 / 4}$ are modified Bessel functions. A graph of $F(\eta)$ is shown in Fig. 1. An important feature in our analysis is that $F(\eta)$ has a maximum,

$$
0 \leq F(\eta) \leq F(\bar{\eta})=1.214 \ldots, \quad \bar{\eta}=0.541 \ldots
$$

The asymptotic properties of $F(\eta)$ follow from (4.11) as

$$
F(\eta) \sim \frac{1}{2 \sqrt{\pi}}\left(1-\frac{\eta^{2}}{2}\right)\left[\Gamma\left(\frac{1}{4}\right)+2 \Gamma\left(\frac{3}{4}\right) \eta\right]+O\left(\eta^{4}\right), \quad \eta \rightarrow 0,
$$

and

$$
F(\eta) \sim \frac{1}{2|\eta|^{1 / 2}} \begin{cases}\sqrt{2} e^{-\eta^{2}}, & \eta \rightarrow-\infty, \\ 1, & \eta \rightarrow \infty .\end{cases}
$$

In view of (4.12), we can conclude from (4.9) that if

$$
\gamma<1 / e F(\bar{\eta})=0.303 \ldots,
$$

then $\hat{\eta}=\infty$ and (4.3) has a bounded solution for $-\infty<\eta<\infty$. Thus, the fulfillment of (4.15) assures that thermal runaway does not occur.

On the other hand, if $\gamma$ is sufficiently large so that

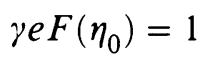

for some $\eta_{0} \leq \bar{\eta}$, then the contraction mapping can only guarantee the existence of a bounded solution to (4.3) for $-\infty<\eta<\eta_{0}$. Thus thermal runaway becomes a 
possibility, and if it does occur as $\eta \rightarrow \eta^{*}$, then we have the bound

$$
F^{-1}(1 / \gamma e)=\eta_{0} \leq \eta^{*} \text {. }
$$

To establish a sufficient condition for the occurrence of thermal runaway as $\eta \rightarrow$ $\eta^{*}$, we employ the following argument. Assume that (4.3) has a continuous and bounded solution on the interval $(-\infty, \tilde{\eta})$, and then seek a condition which constrains the value of $\tilde{\eta}$. We introduce the function

$$
J(\eta)=\gamma \int_{-\infty}^{\eta}[\pi(\tilde{\eta}-\zeta)]^{-1 / 2} \exp \left[u(\zeta)-\zeta^{2}\right] d \zeta, \quad-\infty<\eta<\tilde{\eta} .
$$

From (4.3) it follows that

$$
u(\eta)=\gamma T u(\eta) \geq J(\eta), \quad-\infty<\eta<\tilde{\eta},
$$

and hence

$$
\begin{aligned}
J^{\prime}(\eta) & =\gamma[\pi(\tilde{\eta}-\eta)]^{-1 / 2} \exp \left[u(\eta)-\eta^{2}\right] \\
& \geq \gamma[\pi(\tilde{\eta}-\eta)]^{-1 / 2} \exp \left[J(\eta)-\eta^{2}\right], \quad-\infty<\eta<\tilde{\eta} .
\end{aligned}
$$

Multiplying (4.20) by $\exp [-J(\eta)]$ and integrating over the interval $(-\infty, \tilde{\eta})$ yields

$$
1-\exp [-J(\tilde{\eta})] \geq \gamma F(\tilde{\eta}) \text {. }
$$

If $\gamma$ is sufficiently large so that

$$
\gamma F(\tilde{\eta})=1
$$

for some $\tilde{\eta} \leq \bar{\eta}$, then (4.21) becomes a contradiction of the assumed existence of a continuous solution to (4.3). This implies that thermal runaway has taken place and

$$
\eta^{*} \leq \tilde{\eta}=F^{-1}(1 / \gamma) \text {. }
$$

In view of (4.22) we can conclude that if

$$
\gamma>1 / F(\bar{\eta})=0.823 \ldots,
$$

then thermal runaway does occur as $\eta \rightarrow \eta^{*}$, where

$$
F^{-1}(1 / \gamma e) \leq \eta^{*} \leq F^{-1}(1 / \gamma) \leq \bar{\eta} \text {. }
$$

When thermal runaway does occur, we seek the nature of the singularity experienced by $u(\eta)$ as $\eta \rightarrow \eta^{*}$. After the change of variables

$$
v(\rho)=u(\eta), \quad \rho=1 /\left(\eta^{*}-\eta\right),
$$

(4.3) then takes the form

$$
v(\rho)=\gamma \rho^{1 / 2} \int_{0}^{\rho}\left[\pi(\rho-\omega) \omega^{3}\right]^{-1 / 2} \exp \left[v(\omega)-\left(\eta^{*}-1 / \omega\right)^{2}\right] d \omega .
$$

Now the behavior of $u(\eta)$ as $\eta \rightarrow \eta^{*}$ corresponds to the behavior of $v(\rho)$ as $\rho \rightarrow \infty$. This allows us to utilize the methods of [1] and [2] as analogously applied in [6]. We assume that

$$
\begin{aligned}
v(\rho) & =\log \left\{c_{1} \rho^{1 / 2}+c_{2} \rho^{-1 / 2}+R(\rho)\right\} \\
& =\frac{1}{2} \log \rho+\log c_{1}+\frac{c_{2}}{c_{1}} \rho^{-1}+\cdots, \quad \rho \rightarrow \infty,
\end{aligned}
$$


where the remainder $R(\rho)$ is $O\left(\rho^{-3 / 2}\right)$ as $\rho \rightarrow \infty$. Substitution into the integral of (4.27) gives, as $\rho \rightarrow \infty$,

$$
\begin{aligned}
\gamma \rho^{1 / 2} & \int_{0}^{\rho}\left[\pi(\rho-\omega) \omega^{3}\right]^{-1 / 2} \exp \left[v(w)-\left(\eta^{*}-1 / \omega\right)^{2}\right] d \omega \\
& =\gamma c_{1} e^{-\eta^{* 2}}(\pi)^{-1 / 2} \log \rho+\cdots .
\end{aligned}
$$

A comparison of (4.28) and (4.29) implies that

$$
c_{1}=\sqrt{\pi} e^{\eta^{* 2}} / 2 \gamma .
$$

Thus, as $\eta \rightarrow \eta^{*}$

$$
u(\eta)=-\frac{1}{2} \log \left(\eta^{*}-\eta\right)+\eta^{* 2}-\log (2 \gamma / \sqrt{\pi})+\cdots .
$$

This logarithmic blow-up is similar in form to that found for the solution of (3.22); however, the value of $\eta^{*}$ is different.

The value of $\eta^{*}$ is not determined by the asymptotic analysis, although we have provided bounds in (4.25). An approximate evaluation of $\eta^{*}$ can be obtained by a numerical integration of (4.3), which is matched to (4.31). That evaluation of $\eta^{*}$, which depends on $\gamma$, is shown in Fig. 2.

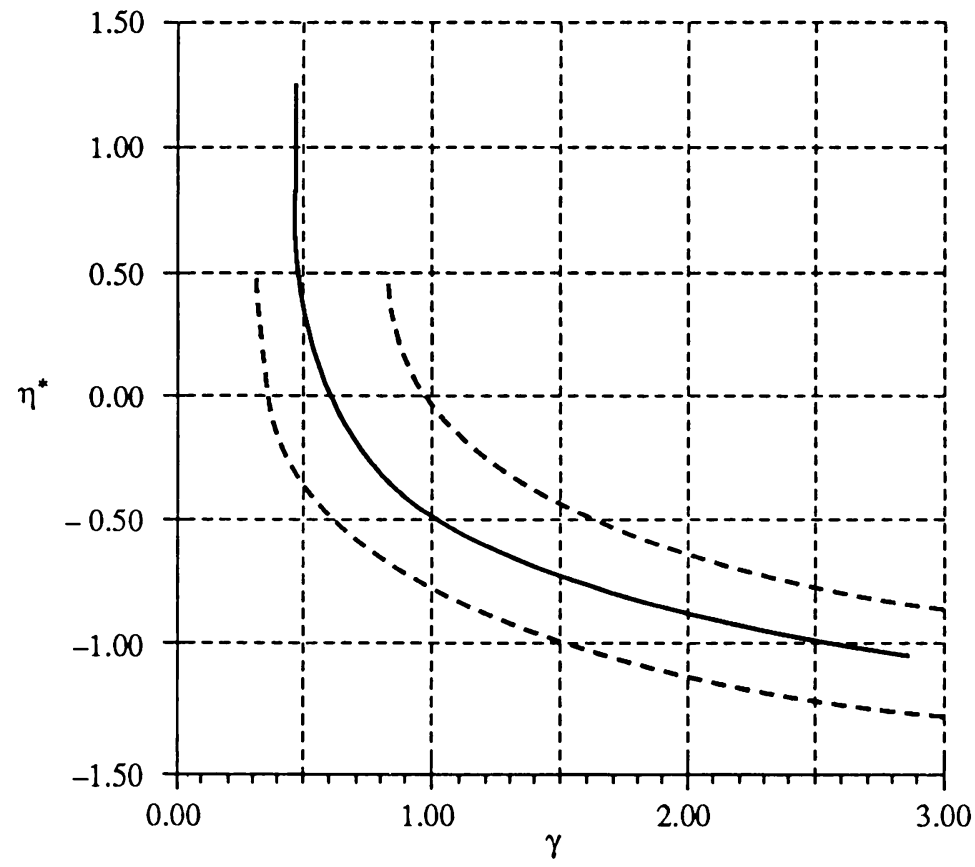

FIG. 2. $\eta^{*}$ vs. $\gamma . \eta^{*}$ is the blow-up time for the solution to (4.3). The dashed lines are the upper and lower bounds on $\eta^{*}$ given by (4.25). 
The asymptotic behavior of $u(\eta)$ as $\eta \rightarrow-\infty$ is found by first noting in (4.3) that $u(\eta) \rightarrow 0$ in this limit. It then follows that

$$
\begin{aligned}
u(\eta) & =\gamma \int_{-\infty}^{\eta}[\pi(\eta-\zeta)]^{-1 / 2}[1+u(\zeta)+\cdots] e^{-\zeta^{2}} d \zeta \\
& =\gamma F(\eta)+\cdots=\frac{\gamma e^{-\eta^{2}}}{\sqrt{2}|\eta|^{1 / 2}}+\cdots, \quad \eta \rightarrow-\infty,
\end{aligned}
$$

where the last equality is obtained from (4.14).

We can also develop an asymptotic solution of (4.3) which is valid for small $\gamma$. Since $\gamma \rightarrow 0$ implies that (4.15) holds, we then know the solution remains bounded and the contraction mapping insures that $u(\eta)$ can be generated by iteration of (4.3). Thus follows

$$
u(\eta)=\gamma F(\eta)+\gamma^{2} \int_{-\infty}^{\eta}[\pi(\eta-\zeta)]^{-1 / 2} e^{-\zeta^{2}} F(\zeta) d \zeta+O\left(\gamma^{3}\right), \quad \gamma \rightarrow 0 .
$$

This expression provides a good approximation of $u(\eta)$ in the case of no thermal runaway.

5. Concluding remarks. We have investigated here the effect of replacing the usual external heating conditions (2.4) by those of marginal heating (2.5). Whereas (2.4) always gives thermal runaway, it is found that $(2.5)$ only produces runaway if $c$ is sufficiently small, while if $c$ is sufficiently large there is no runaway.

In terms of the parameter $\gamma$ defined by (4.2), if (4.15) is satisfied, there is no thermal runaway. On the other hand, if (4.24) is satisfied, runaway occurs as $t \rightarrow t^{*}$, where

$$
t^{*}=t_{0}+(\varepsilon / c)^{1 / 2} \eta^{*}
$$

Here $\eta^{*}=\eta^{*}(\gamma)$ is bounded in (4.25), and its numerically calculated value is given by Fig. 2 .

Acknowledgments. This research was supported in part under NSF grant DMS 8700962. Some of this investigation was done while the second author was a Visiting Associate in Applied Mathematics at the California Institute of Technology under the sponsorship of NSF grant DMS 8706642, AFOSR contract 880269, and ARO contract DAAG 29-85-K-0092.

\section{REFERENCES}

[1] R. A. Handelsman and W. E. Olmstead, Asymptotic solution to a class of Volterra integral equations, SIAM J. Appl. Math. 22, 373-384 (1972)

[2] R. A. Handelsman and W. E. Olmstead, Asymptotic solution to a class of Volterra integral equations. II, SIAM J. Appl. Math. 30, 180-189 (1976)

[3] A. K. Kapila, Evolution of deflagration in a cold combustible subjected to uniform energy flux, Internat. J. Engrg. Sci. 19, 459-509 (1981).

[4] D. G. Lasseigne and W. E. Olmstead, Ignition of a combustible solid with reactant consumption, SIAM J. Appl. Math. 47, 332-342 (1987)

[5] A. Linan and F. A. Williams, Theory of ignition of a reactive solid by constant energy flux, Combustion Sci. Tech. 3, 91-98 (1971)

[6] W. E. Olmstead, Ignition of a combustible half space, SIAM J. Appl. Math. 43, 1-5 (1983) 